\title{
A 12-electrode piezoelectric tube scanner for fast atomic force microscopy
}

\author{
Yuen K. Yong*, Bilal Ahmed and S. O. R. Moheimani \\ School of Electrical Engineering and Computer Science, \\ The University of Newcastle, University Drive, NSW 2308, Australia.
}

\begin{abstract}
This paper presents a 12-electrode piezoelectric tube scanner for fast atomic force microscopy (AFM). The scanner is used simultaneously as a sensor and an actuator. The built-in sensing mechanism of the scanner allows for displacement measurement and the unique arrangement of the electrodes allows the tube to be driven in an anti-symmetrical manner, resulting in a collocated system suitable for positiveposition feedback (PPF). A PPF controller is designed to damp the scanner's resonance. The device is installed into an AFM to obtain open- and closed-loop images of a grating at $10 \mathrm{~Hz}, 15.6 \mathrm{~Hz}$ and $31 \mathrm{~Hz}$ scan rates. The closed-loop images are noticeably superior to the open-loop images, showcasing the effectiveness of the proposed scanner.
\end{abstract}

\section{INTRODUCTION}

Atomic force microscope was invented in the 1980s by Binnig and his collaborators [7]. AFMs are capable of generating three-dimensional maps of material surfaces on an atomic scale. They promise breakthroughs in areas such as material science [14], bio-nanotechnology [2], [20] and nano-indentation for high-density data storage systems [21], [22].

Piezoelectric tube scanners are commonly used in commercially available AFMs to move the sample in the three coordinates. Due to the large length-to-diameter ratio of a tube, when it is fixed at one end its free end will experience a relatively low mechanical resonance frequency. This makes the tube susceptible to scan-induced vibrations. During a typical raster scan, a triangular waveform is applied to the fast axis ( $\mathrm{x}$-axis) and a staircase or ramp signal is applied to the slow axis (y-axis) of the tube. The triangular waveform contains all odd harmonics of the fundamental frequency. The higher harmonic components of a fast triangular waveform (which is a requisite for high-speed scanning) can excite the tube's resonance. To avoid this, the fastest scan rate of an AFM is often limited to $1 \%$ of its scanner's first mechanical resonance frequency [11]. The scan rate of a tube scanner is also limited by non-linearities inherent to the piezoelectric materials, namely hysteresis and creep [8], [15]. The primary objective of this work is to deal with scan-induced vibrations in a AFM scanner and to study its effect on the generated image.

A number of feedback control techniques have been proposed to damp the first resonant mode of piezoelectric tube scanners, and thus to allow for a faster scan. A controller that

\footnotetext{
*Corresponding author. Email: yuenkuan.yong@newcastle.edu.au
}

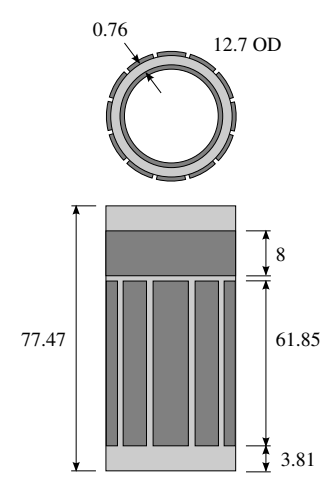

(a)

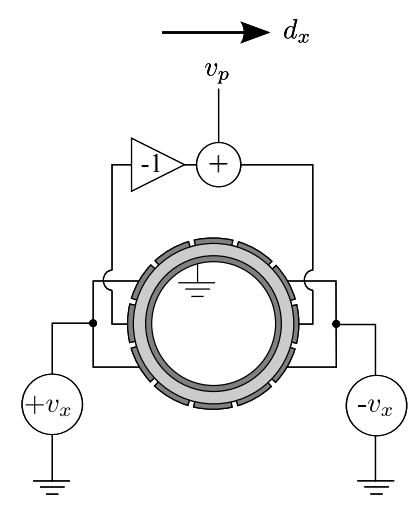

(b)
Fig. 1. Piezoelectric tube scanner. (a) Schematics of the proposed piezoelectric tube scanner with a 12-electrode pattern for $\mathrm{x}$ and $\mathrm{y}$ actuation and sensing. Light areas indicate piezoelectric material, dark areas represent electrodes. All dimensions are in $\mathrm{mm}$. (b) Electrodes wiring for actuation and sensing in the x-direction alone. $v_{x}$ is the applied voltage and $v_{p}$ is the piezoelectric strain-induced voltage. $d_{x}$ represents the displacement measured at the end of the tube. Actuation and sensing in the y direction can be achieved by wiring the appropriate electrodes in a similar way.

is particularly suitable for this purpose is the positive position feedback (PPF) controller [9]. A PPF controller has a simple structure, and thus is easy to implement. Furthermore, when implemented on a collocated system it guarantees closedloop stability. Other vibration control techniques, such as resonant control [19] and integral resonant control [3], [4], [23], are known to provide significant damping to highly resonant systems.

The performance of feedback-based techniques is dependent on the sensor noise and bandwidth. Capacitive sensors (which are commonly used in nanopositioning systems) typically have a RMS noise in the order of $20 \mathrm{pm} / \sqrt{\mathrm{Hz}}$ [11]. This is clearly inadequate for scanning probe microscopy that requires subnanometer precision. Recently, a technique for simultaneous sensing and actuation of a piezoelectric tube was proposed in [5], where one of the tube's quartered electrodes is used for actuation and the opposite electrode is used for sensing. This is a rather attractive proposition since the noise profile of piezoelectric strain-induced voltage has established to be three orders of magnitude lower than a typical capacitive sensor [11]. However, when operated in this mode, (i) the tube is driven asymmetrically, and thus its transfer function deviates from a perfectly collocated system, 
and (ii) the tube's range of motion is reduced by half. Two of the authors recently proposed a new electrode pattern for piezoelectric tube scanners that allows for simultaneous sensing and actuation [17]. The proposed tube scanner avoids the aforementioned complications associated with quartered electrode tubes.

This paper illustrates how the proposed scanner can be modeled and controlled to allow for fast raster scans. Moreover, the controlled scanner is used as the scanning module of a commercial atomic force microscope to obtain highquality images of a calibration grating at $10 \mathrm{~Hz}, 15.6 \mathrm{~Hz}$ and $31 \mathrm{~Hz}$ scan rates. The remainder of the paper is organized as follows. In Sec. II, we describe the proposed electrode pattern and the mechanical design of the scanner. Finite-elementanalysis (FEA) of the tube, simulating its static and dynamic characteristics, is performed in this section. Details of the tube scanner experimental setup is presented in Sec. III. Sec. IV describes the control design, implementation and scan results obtained from the tube. Sec. V concludes the paper.

\section{The 12-electrode Piezoelectric Tube Scanner}

\section{A. Description of the tube scanner and the mechanical design}

Schematics of the 12-electrode piezoelectric tube scanner are shown in Fig. 1. The piezoelectric tube is poled radially outward. The external electrode is segmented into 12 equal sections of $30^{\circ}$ each. It has a small continuous electrode at the top of the tube for $z$-axis actuation. The inside of the tube is covered by a continuous electrode which is grounded at all times. As shown in Fig. 2, one end of the tube scanner is fixed to a base bracket which is mounted to an aluminium casing in order to protect the tube. The assembled tube, base bracket and casing are then mounted on a holder. A sensing target, which also serves as a stage over which a sample can be placed, is glued to the free end of the tube.

The electrode pattern design of the tube is tailored for simultaneous sensing and actuation [17]. Fig. 1(b) illustrates the wiring of the tube for actuation and sensing in the $\mathrm{x}$-axis alone. The wiring of the y-direction is similar and is not illustrated for brevity. The two outer electrodes on opposite sides are used for actuation. When voltages with equal magnitude but opposite polarity are applied to the opposite electrodes, one side of the tube extends and the opposite side retracts, resulting in bending. The strain experienced on each side of the tube is translated into a voltage at the respective central electrode due to the piezoelectric effect. Due to the symmetry, the voltages induced at the opposite electrodes are equal in magnitude but $180^{\circ}$ out of phase. The voltage induced in one electrode is inverted and added to that obtained from the opposite electrode. The resulting signal is then used as an indication of the tube's deflection. Actuation and sensing in the y-direction can be obtained in a similar manner. For z-axis actuation, a voltage is applied to the continuous electrode (z-electrode) near the free end of the tube.

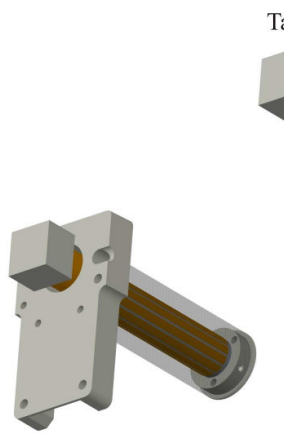

(a)

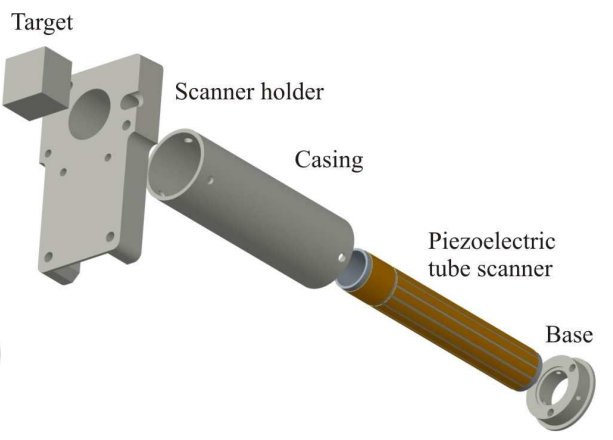

(b)
Fig. 2. CAD drawings of the scanner. (a) Assembly view. (b) Exploded view.

\section{B. Finite-element-analysis of the scanner}

A finite-element (FE) model of the tube scanner was constructed in order to analyze its static and dynamic behavior. The FE model is constructed using three-dimensional elements (SOLID5) in ANSYS. Our objectives are to ensure that the chosen tube scanner provides: (i) a high first resonance frequency for high-speed scanning; (ii) a relatively large displacement range and; (iii) the capability of providing sufficient piezoelectric strain-induced voltages $\left(v_{p}\right)$ for obtaining a good signal-to-noise ratio during measurement. The piezoelectric material PZT-5A is chosen for fabricating the tube scanner and its properties can be found in [1]. The tube dimensions are shown in Fig. 1.

Fig. 3 shows the mode shapes of the tube with a sensing target mounted on top. To simplify the FE model, the simulated results were recorded without incorporating the mass of a sample, sample holder and magnet. There are two identical bending and circumferential modes, each observed in the $\mathrm{x}$ and $\mathrm{y}$ axes respectively. For brevity, only modes corresponding to the $\mathrm{x}$-axis are shown in Fig. 3. The first resonant mode is the bending mode occurring at $658.4 \mathrm{~Hz}$. Torsional, longitudinal and circumferential modes of the tube are also observed.

Assuming the system is linear, the static (DC) gain of the tube is estimated to be $d_{x} / v_{x}=0.0724 \mu \mathrm{m} / V$. The sensitivity of the tube is estimated to be $v_{p_{x}} / d_{x}=0.2 \mathrm{~V} / \mu \mathrm{m}$. When an input voltage of $300 \mathrm{~V}$ is applied, the tube is displaced by $21.7 \mu \mathrm{m}$. This amounts to $v_{p_{x}}=4.3 \mathrm{~V}$, providing a good measurement signal for sensing. Gains of both the $\mathrm{x}$ and $\mathrm{y}$ axes are the same due to the symmetry. For z-axis actuation, the static (DC) gain of the tube is estimated to be $d_{z} / v_{z}=1.97 \mathrm{~nm} / \mathrm{V}$, where $v_{z}$ is the voltage applied to the z-electrode. The tube provides a z-displacement of $0.6 \mu \mathrm{m}$ when $300 \mathrm{~V}$ is applied to this electrode. The $\mathrm{z}$ range is sufficient for atomic force microscopy.

\section{System Description ANd Experimental SetuP}

Fig. 4 shows the NT-MDT NTEGRA scanning probe microscope (SPM) used to perform experiments reported here. The SPM is configured to operate as an AFM. The original scanner of the SPM was replaced by the proposed 


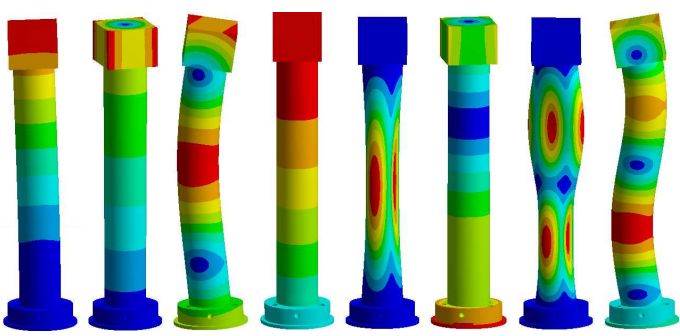

Fig. 3. ANSYS simulated mode shapes of the scanner (without sample mass). The casing of the scanner is hidden in order to display the mode shapes clearly. From the left, first bending mode: $658.4 \mathrm{~Hz}$; first torsional mode: $4.06 \mathrm{kHz}$; second bending mode: $4.65 \mathrm{kHz}$; first longitudinal extension mode: $5.76 \mathrm{kHz}$; first circumferential mode: $9.25 \mathrm{kHz}$; second torsional mode: $10.51 \mathrm{kHz}$; second circumferential mode: $10.77 \mathrm{kHz}$; third bending mode: $10.9 \mathrm{kHz}$.

tube scanner (see Fig. 4(b)). A protective hood was used as a shield against acoustic noise, electromagnetic fields and temperature variations. An aluminium ring was designed to serve as a base over which the protective hood was placed (see Fig. 4(a)). A feedthrough connector was mounted into the ring to serve as a signal access unit to the scanner. This setup allows one to by-pass the hood and to gain direct access to the electrodes of the scanner. The modified AFM system allows the tube scanner to be driven by external voltage amplifiers. This modification also enabled us to implement a damping controller (to improve tube's resonant behavior) externally without the need for modifying the existing control logic of the AFM system.

The $\mathrm{x}$ and $\mathrm{y}$ axes of the tube were driven by a NANONIS bipolar high voltage amplifier. Piezoelectric strain-induced voltages have a first-order high-pass characteristic at low frequencies. This is due to the capacitive nature of the sensors and finite input impedance of the measurement device. To minimize this characteristic, voltages measured at the central electrodes of the tube were fed to two low noise preamplifiers (Stanford Research Systems SR560). The input impedance of the preamplifier is $100 \mathrm{M} \Omega$. The measured capacitance of each electrode is $3.8 \mathrm{nF}$ [17]. Together with the input impedance of $100 \mathrm{M} \Omega$ of the preamplifier, the cut-off frequency of the sensing signal is reduced to less than $1 \mathrm{~Hz}$. Two ADE Technologies 8810 capacitive sensors were placed in close proximity to the adjacent surfaces of the sample holder to observe the displacements of the tube along the $\mathrm{x}$ and $y$ axes (see Fig. 4(b)).

The AFM controller was used to generate the $\mathrm{x}$ and $\mathrm{y}$ signals. These were accessed through the AFM signal access module and were applied to the controlled piezoelectric tube scanner through the high voltage amplifiers. A dSPACE1103 rapid prototyping system was used to implement the $\mathrm{x}$ and $\mathrm{y}$ axes feedback controllers in real-time. The $\mathrm{z}$-axis displacement was controlled using the AFM's software and circuitry.

To design a controller for the scanner, one requires a dynamic model of the device. Such a model can be identified from the frequency response functions (FRFs) obtained from the apparatus. The FRFs of the scanner were obtained using a

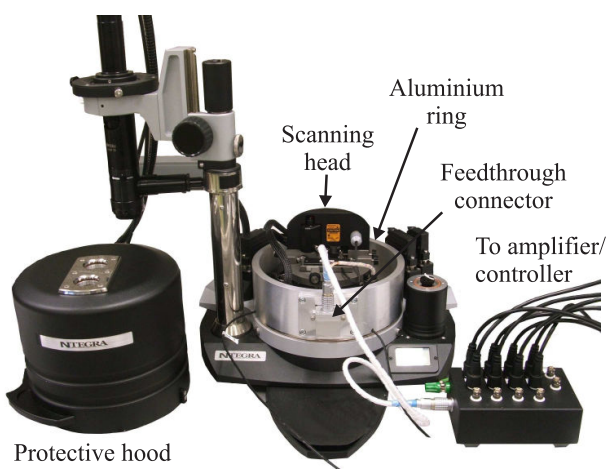

(a)

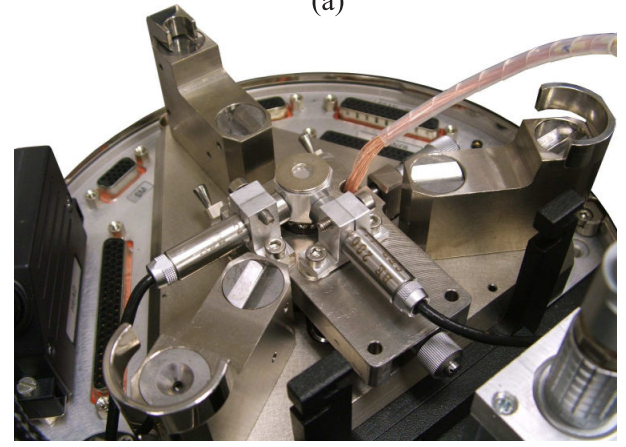

(b)

Fig. 4. Experimental apparatus. (a) A modified NT-MDT NTEGRA SPM. The tube scanner is located below the scanning head. (b) The tube scanner is installed into the SPM. The two capacitive sensors are mounted at right angles to the target.

bandlimited swept sine input of amplitude $200 \mathrm{mVpk}$, within the frequency range of $10 \mathrm{~Hz}$ to $1 \mathrm{kHz}$, and using a $\mathrm{HP}$ $35670 \mathrm{~A}$ dual channel spectrum analyzer. In this case, the scanner was treated as a multivariable system. The two inputs are the voltages applied to the $\mathrm{x}$ and $\mathrm{y}$ axes amplifiers $\left[v_{x}, v_{y}\right]^{T}$ while the outputs are the corresponding straininduced voltages $\left[v_{p_{x}}, v_{p_{y}}\right]^{T}$, and displacements $\left[d_{x}, d_{y}\right]^{T}$ of the tube measured by the capacitive sensors. The two measured subsystems are $G_{v v}(j \omega)=\left[\begin{array}{ll}G_{x x}(j \omega) & G_{x y}(j \omega) \\ G_{y x}(j \omega) & G_{y y}(j \omega)\end{array}\right]$ and $G_{d v}(j \omega)=\left[\begin{array}{ll}G_{d_{x} x}(j \omega) & G_{d_{x} y}(j \omega) \\ G_{d_{y} x}(j \omega) & G_{d_{y} y}(j \omega)\end{array}\right]$. The subscript $v v$ in $G_{v v}(j \omega)$ denotes that both the inputs and outputs are voltages, whilst the subscript $d v$ in $G_{d v}(j \omega)$ denotes that the inputs are voltages and outputs are displacements.

The measured open-loop FRFs of $G_{v v}$ and $G_{d v}$ are shown in Fig. 6. It can be observed that the two transfer functions $G_{v v}(s)$ and $G_{d v}(s)$ have identical poles. Therefore, a controller which provides the damping of $G_{v v}(s)$ will have a similar effect on $G_{d v}(s)$ [17]. The FRFs were captured with a total mass of $4.3 \mathrm{~g}$ (i.e., the total mass of a sample, sample holder and magnet) mounted on top of the tube. The first resonant peak appears at $499 \mathrm{~Hz}$, which is $160 \mathrm{~Hz}$ less than the FE simulated result due to the additional mass. For comparison purposes, a small rectangular block with an equivalent mass of $4.3 \mathrm{~g}$ was incorporated into the FE model. This FE model predicts that the first resonant mode would appear at $530 \mathrm{~Hz}$, which is about $6 \%$ different from 
the experimental outcome. The measured sensitivities of the tube $v_{p_{x}} / d_{x}=0.17 \mathrm{~V} / \mu \mathrm{m}, d_{x} / v_{x}=0.0728 \mu \mathrm{m} / \mathrm{V}, d_{z} / v_{z}=$ $2.1 \mathrm{~nm} / \mathrm{V}$, are in close agreement with the FE simulations in Sec. II.

\section{CONTROL Design AND IMPlementation}

\section{A. Control design}

The main objective of this section is to design a feedback controller which will provide significant damping to $G_{d_{x} x}$ by using $v_{p_{x}}$ as measurement signal. A positive position feedback (PPF) controller was designed and implemented to damp the first resonant mode of $\mathrm{x}$ and $\mathrm{y}$ axes. The block diagram of the closed-loop system is shown in Fig. 5. The control scheme uses the induced voltages $v_{p_{x}}$ as the measurement for the PPF controller, while the displacement $d_{x}$ is observed using the capacitive sensor.

For brevity, only the X-axis controller design is discussed here. A controller for the y-axis was designed along similar lines. A $2^{\text {nd }}$ order model was fitted to the FRF data corresponding to $G_{x x}$ (using the frequency domain subspace modeling technique [13]) to accurately capture the first dominant peak of the tube. The transfer function of the model obtained is $G_{\text {model }}(s)=$ $\left[8.065 \times 10^{6} /\left(s^{2}+68.41 s+9.75 \times 10^{6}\right)\right]-0.6643$. Transfer function of $G_{\text {model }}(s)$ can be written in time domain form as follows [6]:

$$
\begin{gathered}
\ddot{x}+2 \sigma \omega \dot{x}+\omega^{2} x=\psi_{1} u \\
y=\psi_{2} x+d u
\end{gathered}
$$

where $\psi_{1}=8.0657 \times 10^{6}, \psi_{2}=1, d=-0.6643, \sigma=$ $1.095 \times 10^{-2}$, and $\omega=3.1225 \times 10^{3}$.

The control objective is to design a control law $u$ for the system in Eq. (1) such that the closed-loop FRF would imply a well damped system. To achieve this objective, PPF control design technique [9] is used in this paper. The controller is defined as follows:

$$
\ddot{z}+2 \zeta \eta \dot{z}+\eta^{2} z=\Gamma v_{p_{x}}
$$

where $v_{p_{x}}$ is the input to the controller, and $\zeta, \eta, \Gamma$ are the design parameters.

The input $u$ in Eq. (1) is defined by $u(t) \triangleq z+r$ where $r(t)$ is the reference signal and $z(t)$ is the controller state. Substituting this in Eq. (1) we obtain

$$
\begin{gathered}
\ddot{x}+2 \sigma \omega \dot{x}+\omega^{2} x=\psi_{1}(z+r), \\
y=\psi_{2} x+d(z+r) .
\end{gathered}
$$

The controller input in Eq. (2) is set to $v_{p x}(t) \triangleq y(t)$. Let $\rho \triangleq\left(\eta^{2}-\Gamma d\right)$, Eq. (2) reduces to

$$
\ddot{z}+2 \zeta \eta \dot{z}+\rho z=\Gamma\left(\psi_{2} x+d r\right) .
$$

Taking the Laplace transform of Eqs. (3) and (4) we get

$$
\begin{gathered}
{\left[\begin{array}{cc}
s^{2}+2 \sigma \omega s+\omega^{2} & -\psi_{1} \\
-\Gamma \psi_{2} & s^{2}+2 \zeta \eta s+\rho
\end{array}\right]\left[\begin{array}{l}
x(s) \\
z(s)
\end{array}\right]} \\
=\left[\begin{array}{l}
\psi_{1} \\
\Gamma d
\end{array}\right] r(s), \\
y(s)=\left[\begin{array}{ll}
\psi_{2} & d
\end{array}\right]\left[\begin{array}{l}
x(s) \\
z(s)
\end{array}\right]+d r(s) .
\end{gathered}
$$

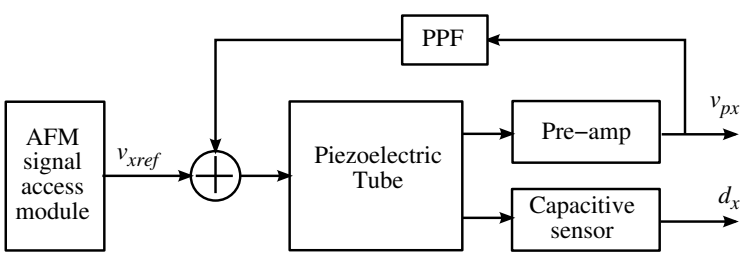

Fig. 5. Block diagram of the $x$-axis closed-loop system. The y-axis closedloop system is similar. $v_{x_{r e f}}$ is the scanning reference waveform provided by the AFM signal access module. The output $v_{p_{x}}$ is used as an input to the controller. The output $d_{x}$ is the displacement of the tube scanner.

The closed loop poles of system in Eq. (5) are the roots of polynomial equation given by $P(s) \triangleq s^{4}+(2 \zeta \eta+2 \sigma \omega) s^{3}+$ $\left(\rho+4 \sigma \omega \zeta \eta+\omega^{2}\right) s^{2}+\left(2 \sigma \omega \rho+2 \zeta \eta \omega^{2}\right) s+\rho \omega^{2}-\Gamma \psi_{1} \psi_{2}$. The PPF controller is designed to place the poles $\left\{P_{i}\right\}_{i=1}^{4}$ of Eq. (5) in a desired region of the left half plane. This design is achieved by choosing unknown parameters $(\zeta, \eta, \Gamma)$ of $P(s)$ such that $V=\sum_{k=1}^{4}\left|P_{k}^{d}-\operatorname{Re}\left(P_{k}^{c}\right)\right|^{2}$ is minimal [16]. The cost function $V$ minimizes the difference between the real part of the closed-loop poles $\left\{P_{k}^{c}\right\}^{4}$ and the set of prespecified real values $\left\{P_{k}^{d}\right\}^{4}$ in the left half plane.

Experiments were conducted on the scanner to demonstrate the practical application of the proposed controller. The PPF controller was connected with the outputs $v_{p_{x}}, v_{p_{y}}$ to the inputs $v_{x}, v_{y}$ to damp the resonance in the systems $G_{x x}, G_{y y}$, respectively. The experimental results are discussed below.

Open-loop poles of the transfer function $G_{\text {model }}(s)$ are given by $P \pm=-34.2 \pm$ i $3.12 \times 10^{3}$.

The PPF controller was designed in MATLAB/SIMULINK environment. The desired closed-loop pole locations were set further into the left half plane to impart sufficient damping in the closed-loop system. MATLAB function lsqnonlin was used to solve the optimization problem. The actual closed-loop poles were found to be $P_{1+}^{c}=P_{2+}^{c}=-1.01 \times 10^{3} \pm \mathrm{i} 2.31 \times 10^{3}$, $P_{1-}^{c}=P_{2-}^{c}=-6.21 \times 10^{2} \pm \mathrm{i} 3.39 \times 10^{3}$. Solving for the controller parameters $\zeta, \eta, \Gamma$ from Eq. (2) using the cost function $V$, we obtain the PPF controller

$$
K_{P P F}(s) \triangleq \frac{3.663 \times 10^{6}}{s^{2}+3202 s+8.337 \times 10^{6}} .
$$

Fig. 6 shows the measured open- and closed-loop FRFs of $G_{v v}(j \omega)$ and $G_{d v}(j \omega)$. It is clear that the proposed controller for the system $G_{v v}$ provides a similar effect on $G_{d v}$. It is evident from Fig. 6(b) that a damping of $21 \mathrm{~dB}$ is achieved at the dominant resonant mode using the PPF controller. The controller does not disturb the high frequency dynamics of the system. Furthermore, the experimental results show a significant damping in the cross-coupling terms.

\section{B. Scan Results}

In this section, scanned images of a calibration grating are used to evaluate the closed-loop performance of the tube scanner. A MikroMasch TGQ1 calibration grating with $3 \mu \mathrm{m}$ period, $1.5 \mu \mathrm{m}$ square side and $20 \mathrm{~nm}$ height was used for experiments. A contact mode ContAl cantilever probe with 

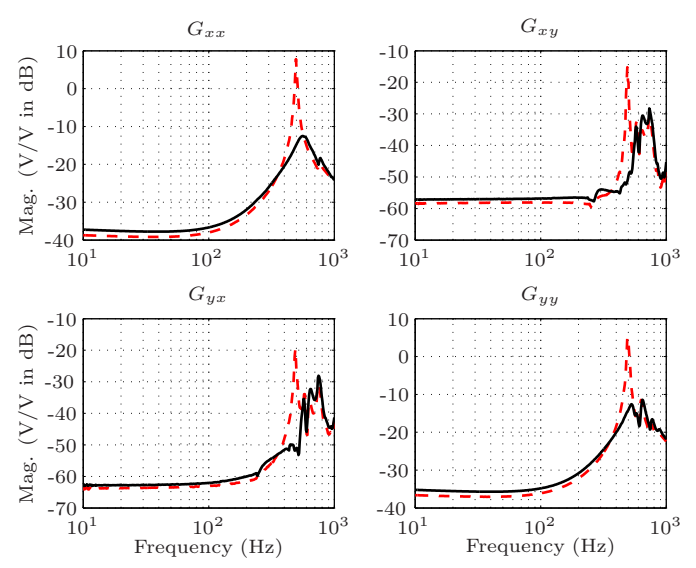

(a)
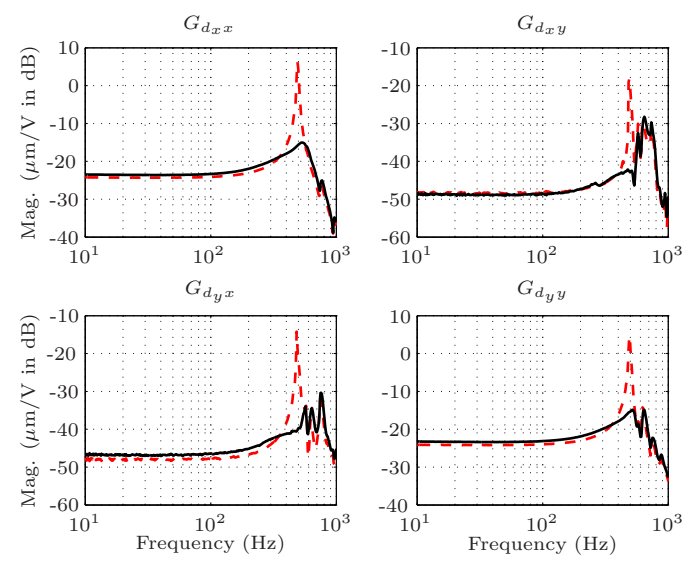

(b)

Fig. 6. Measured open- (- -) and closed-loop (-) gain response of $G_{v v}$ (a) and $G_{d v}$ (b).

a resonance frequency of $13 \mathrm{kHz}$ was used to perform the scan. The vibration of the $\mathrm{x}$ and $\mathrm{y}$ axes are damped using the PPF controller. The $\mathrm{z}$-axis displacement is controlled by the AFM's in-built controller and voltage amplifier. $10 \mu \mathrm{m} \times$ $10 \mu \mathrm{m}$ images (with $256 \times 256$ scan lines) of the grating were recorded in constant height contact mode, in both open- and closed-loop at $10 \mathrm{~Hz}, 15.6 \mathrm{~Hz}$ and $31 \mathrm{~Hz}$ scan rates.

Fig. 7 compares the open- and closed-loop images obtained from the modified AFM. At $10 \mathrm{~Hz}$, the oscillation is visible in both the image and the measured $\mathrm{x}$-axis displacement. The oscillation is suppressed when the PPF controller is activated.

Vibrations severely deteriorate the quality of the images at $15.6 \mathrm{~Hz}$ and $31 \mathrm{~Hz}$. The scanner vibrates at a higher magnitude at $31 \mathrm{~Hz}$ than at $10 \mathrm{~Hz}$ and $15.6 \mathrm{~Hz}$, but the image does not appear to be much more distorted. This is due to the fact that the magnitude of oscillation at $31 \mathrm{~Hz}$ is almost the same as the square side length of the image. With the controller activated, the oscillations are again eliminated.

The $\mathrm{x}$ and $\mathrm{y}$ axes were driven using a voltage amplifier and images were generated over a relatively large scan range. The hysteresis effect is minor but rather noticeable in both the images and measured $\mathrm{x}$-axis displacements. Feedforward techniques, which do not require an on-line sensor, can be used to reduce the hysteresis effect [15]. A number of accurate hysteresis models, such as the Preisach models [18] and the Maxwell resistive capacitor model [12], can be utilized in conjunction with feedforward controls to minimize the presence of hysteresis. Alternatively, the scanner can be driven by charge amplifiers, which are known to linearize the piezoelectric actuators [10], [23].

\section{Conclusions}

The use of a new type of piezoelectric tube scanner, with simultaneous sensing and actuation capabilities, for an atomic force microscopy was studied in this paper. A FE model of the scanner was constructed in ANSYS to analyze its static and dynamic behavior. The obtained FE model suggests a good agreement with the experimental outcomes. A dynamic model of the scanner was then identified and, based on this model, a PPF controller was designed and implemented to improve its resonant behavior. Scan images of $10 \mu \mathrm{m} \times 10 \mu \mathrm{m}$ were generated with the scanner operated both in open- and closed-loop, at $10 \mathrm{~Hz}, 15.6 \mathrm{~Hz}$ and $31 \mathrm{~Hz}$. Closed-loop images were observed to be much more superior compared to the open-loop images, implying the effectiveness of using strain-induced voltages in an AFM feedback system.

\section{ACKNOWLEDGEMENT}

This research was supported by the Australian Research Council Centre of Excellence for Dynamic Systems and Control.

\section{REFERENCES}

[1] see Boston Piezo-Optics http://bostonpiezooptics.com for piezoceramic material properties.

[2] T. Ando, N. Kodera, D. Maruyama, E. Takai, K. Saito, and A. Toda, "A high-speed atomic force microscope for studying biological macromolecules in action," Japanese Journal of Applied Physics, vol. 41, no. 7B, pp. 4851-4856, 2002.

[3] S. S. Aphale, A. J. Fleming, and S. O. R. Moheimani, "Integral resonant control of collocated smart structures," Smart Materials and Structures, vol. 16, pp. 439-446, 2007.

[4] B. Bhikkaji and S. O. R. Moheimani, "Integral resonant control of a piezoelectric tube actuator for fast nano-scale positioning," IEEE/ASME Trans. on Mechatronics, vol. 13, no. 5, pp. 530-537, October 2008.

[5] B. Bhikkaji, M. Ratnam, A. J. Fleming, and S. O. R. Moheimani, "High-performance control of piezoelectric tube scanners," IEEE Trans. Contr. Syst. Tech., vol. 15, no. 5, pp. 853-866, 2007.

[6] B. Bhikkaji, M. Ratnam, and S. O. R. Moheimani, "PVPF control of piezoelectric tube scanners," Sensors and Actuators A, vol. 135, pp. 700-712, 2007.

[7] G. Binnig, C. F. Quate, and C. Gerber, "Atomic force microscope," Phys. Rev. Lett., vol. 56, pp. 930-933, 1986.

[8] D. Croft, G. Shedd, and S. Devasia, "Creep, hysteresis, and vibration compensation for. piezoactuators: Atomic force microscopy application," Journal of Dynamic Systems, Measurement, and Control, vol. 123, no. 1, pp. 35-43, 2001.

[9] J. L. Fanson and T. K. Caughey, "Positive position feedback control for large space structures," AIAA Journal, vol. 28, no. 4, pp. 717 $724,1990$.

[10] A. J. Fleming and S. O. R. Moheimani, "A grounded-load charge amplifier for reducing hysteresis in piezoelectric tube scanners," Review of Scientific Instruments, vol. 76, no. 7, p. 073707, 2005.

[11] A. J. Fleming, A. Wills, and S. O. R. Moheimani, "Sensor fusion for improved control of piezoelectric tube scanners," IEEE Trans. Contr. Syst. Tech., vol. 16, no. 6, pp. 1265-1276, November 2008. 

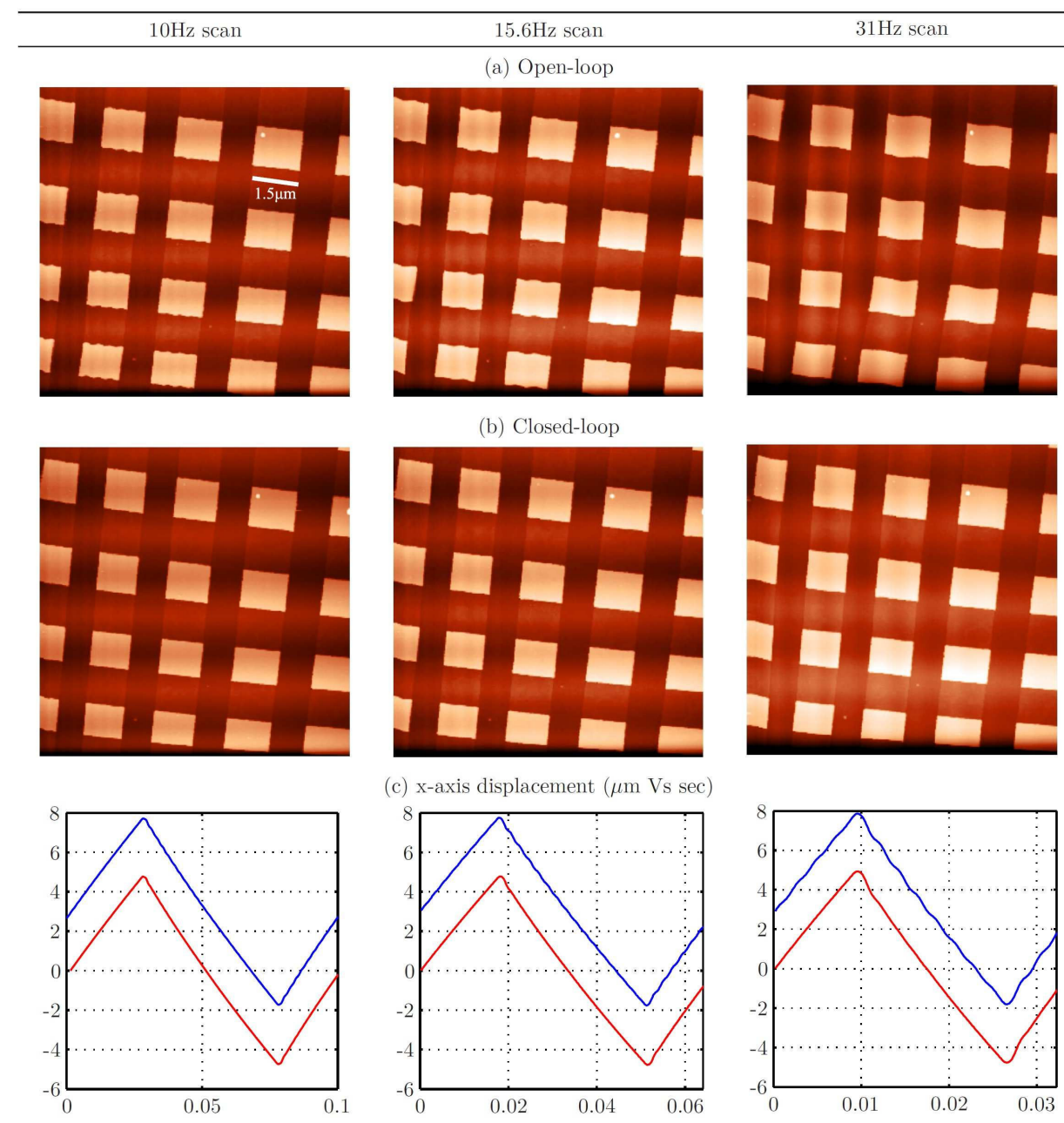

Fig. 7. Recorded images $(10 \mu \mathrm{m} \times 10 \mu \mathrm{m})$ of the grating at $10 \mathrm{~Hz}, 15.6 \mathrm{~Hz}$ and $31 \mathrm{~Hz}$. (a) Oscillations are clearly visible in the three open-loop scans. (b) Vibrations are eliminated in the closed-loop images. Artifacts at the edge of closed-loop scans are due to the limited bandwidth of the feedback system. (c) Open- (with a positive offset) and closed-loop triangular waveforms are plotted. The bandwidth limitation effect of the closed-loop system is clearly noticeable at $31 \mathrm{~Hz}$.

[12] M. Goldfarb and N. Celanovic, "A lumped parameter electromechanical model for describing the nonlinear behavior of piezoelectric actuators," Journal of Dynamic Systems, Measurement, and Control, vol. 119 , no. 3, pp. 478-485, 1997.

[13] T. McKelvey, H. Akcay, and L. Ljung, "Subspace based multivariable system identification from frequency response data," IEEE Trans. on Automatic Control, vol. 41, no. 7, pp. 960-978, July 1996.

[14] K. Miyahara, N. Nagashima, T. Ohmura, and S. Matsuoka, "Evaluation of mechanical properties in nanometer scale using AFM-based nanoindentation tester," Nanostructured Materials, vol. 12, no. 5-8, pp. 1049 - 1052, 1999, the Fourth International Conference on Nanostructured Materials (NANO '98).

[15] S. O. R. Moheimani, "Invited review article: Accurate and fast nanopositioning with piezoelectric tube scanners: Emerging trends and future challenges," Review of Scientific Instruments, vol. 79, no. 7, p. 071101, July 2008, article Number: 071101.

[16] S. O. R. Moheimani, B. J. G. Vautier, and B. Bhikkaji, "Experimental implementation of extended multivariable PPF control on an active structure," IEEE Trans. Contr. Syst. Tech., vol. 14, no. 3, pp. 443-455, 2006.

[17] S. O. R. Moheimani and Y. K. Yong, "Simultaneous sensing and actuation with a piezoelectric tube scanner," Review of Scientific Instruments, vol. 79, no. 7, July 2008, article Number: 073702.

[18] R. B. Mrad and H. Hu, "A model for voltage-to-displacement dynamics in piezoceramic actuators subject to dynamic-voltage excitations," Mechatronics, IEEE/ASME Transactions on, vol. 7, no. 4, pp. 479489, Dec. 2002
[19] H. R. Pota, S. O. R. Moheimani, and M. Smith, "Resonant controllers for smart structures," Smart Materials and Structures, vol. 11, no. 1, pp. 1-8, 2002.

[20] M. J. Rost, G. J. C. van Baarle, A. J. Katan, W. M. van Spengen, P. Schakel, W. A. van Loo, T. H. Oosterkamp, and J. W. M. Frenken, "Video-rate scanning probe control challenges: setting the stage for a microscopy revolution," Asian Journal of Control, vol. 11, no. 2, pp. $110-129,2009$.

[21] A. Sebastian, A. Pantazi, S. O. R. Moheimani, H. Pozidis, and E. Eleftheriou, "Achieving sub-nanometer precision in a MEMS storage device during self-servo write process," IEEE Transactions on Nanotechnology, vol. 7, no. 5, pp. 586-595, 2008.

[22] P. Vettiger, G. Cross, M. Despont, U. Drechsler, U. Dürig, B. Gotsmann, W. Häberle, M. A. Lantz, H. E. Rothuizen, R. Stutz, and G. K. Binnig, "The "Milipede" - nanotechnology entering data storage," IEEE Trans. Nanotechnology, vol. 1, no. 1, pp. 39-55, 2002.

[23] Y. K. Yong, S. Aphale, and S. O. R. Moheimani, "Design, identification and control of a flexure-based XY stage for fast nanoscale positioning," IEEE Trans. Nanotechnology, vol. 8, no. 1, pp. 46-54, 2009. 